\title{
Sustained and Controlled Release of Daunomycin from Cross-Linked Poly(aldehyde guluronate) Hydrogels
}

\author{
KAMAL H. BOUHADIR, ${ }^{1,3}$ GENEVEIVE M. KRUGER, ${ }^{2}$ KUEN YONG LEE, ${ }^{1,3}$ DAVID J. MOONEY ${ }^{1,2,3}$ \\ ${ }^{1}$ Department of Chemical Engineering, University of Michigan, 3074 H. H. Dow Bldg., 2300 Hayward Street, \\ Ann Arbor, Michigan 48109-2136 \\ 2 Department of Biomedical Engineeering, University of Michigan, Ann Arbor, Michigan 48109 \\ ${ }^{3}$ Department of Biological \& Materials Sciences, University of Michigan, Ann Arbor, Michigan 48109
}

Received 23 June 1999; revised 16 February 2000; accepted 29 February 2000

\begin{abstract}
We have incorporated daunomycin, an antineoplastic agent, into a biodegradable hydrogel through a labile covalent bond. In brief, sodium alginate was chemically broken down to low molecular weight and followed by oxidation to prepare poly(aldehyde guluronate). Adipic dihydrazide was used to incorporate the drug into the polymer backbone and cross-link the polymer to form hydrogels. Daunomycin can be released from the hydrogel after the hydrolysis of the covalent linkage between the drug and the polymer. A wide range of release profiles of daunomycin (e.g., from 2 days to 6 weeks) has been achieved using these materials, and the biological activity of the released daunomycin was maintained. (C) 2000 Wiley-Liss, Inc. and the American Pharmaceutical Association J Pharm Sci 89: 910-919, 2000

Keywords: hydrogel; poly(aldehyde guluronate); daunomycin; covalent cross-linking; controlled delivery
\end{abstract}

\section{INTRODUCTION}

One common approach in the treatment of cancer is the delivery of antineoplastic agents to the tumor. However, to achieve complete eradication of tumors, antineoplastic agents must be administered in high doses intravenously, which unfortunately leads to the killing of healthy cells in addition to the cancer cells. Adverse side effects can include severe immunosuppression, myelosuppression, nephrotoxicity, and cardiotoxicity. ${ }^{1,2}$ In theory, the localized delivery of potent agents at the required concentration should eradicate cancer cells without causing any damage to healthy tissues and organs. This method may then allow one to deliver antineoplastic agents in a controlled manner over a wide range of time frames depending on the type, size, and growth rate of

Correspondence to: D. J. Mooney (Telephone: 734-7634816; Fax: 734-763-0459; E-mail: mooneyd@umich.edu)

Journal of Pharmaceutical Sciences, Vol. 89, 910-919 (2000)

(C) 2000 Wiley-Liss, Inc. and the American Pharmaceutical Association the tumor. There have been many attempts to deliver antineoplastic agents using various delivery systems to circumvent adverse side effects and to enhance the efficiency of the drugs. ${ }^{3-12}$

Hydrogels have been widely investigated as delivery vehicles for the localized, sustained release of bioactive agents. ${ }^{13,14}$ Many synthetic and naturally derived materials have been reported to form hydrogels, ${ }^{15-18}$ and one widely used hydrogel is formed from the ionic cross-linking of sodium alginate, a linear polysaccharide isolated from seaweed. Alginate is comprised of $(1,4)$ linked $\beta$-D-mannuronic and $\alpha$-L-guluronic acid residues arranged in blocks of polymannuronate, polyguluronate, and alternating units of both sugars. Divalent cations, such as calcium, ionically cross-link the carboxylate groups on adjacent alginate strands to form hydrogels. The polyguluronate block of alginate is known to be responsible for this gelling feature. ${ }^{19}$ The favorable properties of alginate, including nonimmunogenicity, hydrophilicity, and relatively low cost have prompted 
attempts to use this material as wound dressing, dental impression, and immobilization scaffolds for cultured and transplanted cells. ${ }^{20-22}$ Alginate is considered to be a biocompatible polymer, ${ }^{23}$ although contaminating factors may induce significant inflammation if the polymer is not suitably purified. ${ }^{24}$ Alginate hydrogels have been previously proposed for a number of drug delivery applications. ${ }^{25-27}$ One disadvantage of alginate hydrogels is that they are not chemically broken down in mammals. They instead dissolve in an uncontrollable and unpredictable manner following the dissolution of calcium into the surrounding medium. Furthermore, the molecular weight of intact alginate is typically above the renal clearance threshold, thus preventing it from being excreted from the body. ${ }^{28}$

To overcome these disadvantages, we have recently synthesized new hydrogels composed of low molecular weight polymers derived from alginate. ${ }^{29}$ In brief, alginate was hydrolyzed under acidic conditions and the polyguluronic acid blocks were isolated, transformed to sodium polyguluronate, and oxidized to yield poly(aldehyde guluronate) (PAG). PAG was then crosslinked with adipic dihydrazide to form biodegradable hydrogels with enhanced and easily controlled mechanical properties. We now report the utilization of these hydrogels to deliver an antineoplastic agent, daunomycin, in a controlled manner over a wide range of time frames. Daunomycin was covalently attached to PAG via a dihydrazide spacer, resulting in the formation of hydrazone bonds between the polymer and the daunomycin. The drug was released from the hydrogel by the hydrolysis of the hydrazone bond. The release of daunomycin from the hydrogel was monitored in vitro and was controlled by varying the amount of covalent or ionic cross-linking of the hydrogels. The bioavailability of the released drug was tested in vitro as well.

\section{EXPERIMENTAL SECTION}

\section{Materials}

Sodium alginate was purchased from Pronova Biomaterials (Drammen, Norway). Sodium periodate, adipic dihydrazide, ethylene glycol, and calcium chloride were purchased from Aldrich Chemical Company (Milwaukee, WI) and were used as received. Daunomycin hydrochloride was purchased from Fluka Chemical Corporation (Ronkonkoma, NY). Dulbecco's Modified Eagle's
Medium (DMEM) was purchased from Life Technologies (Grand Island, NY). KB cells (an established line of human epidermoid carcinoma cells) were a gift from Dr. John Drach (Department of Biologic \& Materials Sciences, University of Michigan).

\section{Instruments}

The ultraviolet/visible (UV/vis) spectra were collected on a Perkin Elmer Lambda 12 UV/VIS spectrophotometer. High-performance liquid chromatography (HPLC) analysis was performed with a Hewlett Packard Series II 1090 liquid chromatograph with a photodiode array detector and a Phenomenex Hypersil C18 column. The system was programmed for gradient elution starting with $82 / 18$ ([0.025\% aqueous acetic acid]/ $\mathrm{CH}_{3} \mathrm{CN}$, v/v) for 2 min then raised to 50/50 during 4 min then back to $82 / 18$. The elution of the drug was monitored at $480 \mathrm{~nm}$ wavelength.

\section{Synthesis of Daunomycin-Adipoyl Hydrazide Hydrochloride}

A 500-mL round-bottomed flask was wrapped with aluminum foil and charged with daunomycin hydrochloride $(25 \mathrm{mg}, 44.3 \mu \mathrm{mol})$ and adipic dihydrazide (434 mg, $2.49 \mathrm{mmol}$ ). Methanol (250 $\mathrm{mL}$ ) was added, and the reaction was stirred at room temperature in the dark for 5 days. The solvent was concentrated to $50 \mathrm{~mL}$ under reduced pressure. The precipitated adipic dihydrazide was removed by filtration, and the filtrates were concentrated to $5 \mathrm{~mL}$ under reduced pressure. Chloroform $(5 \mathrm{~mL})$ was added in a dropwise manner while shaking, and the mixture was cooled at $4{ }^{\circ} \mathrm{C}$ overnight. More adipic dihydrazide precipitated. The solvent was carefully transferred into a 25$\mathrm{mL}$ scintillation vial with a Pasteur pipette, leaving the precipitate behind. The solvent was evaporated under reduced pressure to yield $25.2 \mathrm{mg}$ of product (35 $\mu \mathrm{mol}, 80 \%$ yield) that was analyzed by thin-layer chromatography (TLC) on Kodak 13181 silica gel in $\mathrm{CHCl}_{3} / \mathrm{CH}_{3} \mathrm{OH}$ (80/20) (daunomycin hydrochloride: $R_{f}=0.39$; daunomycinadipoyl hydrazide: $R_{f}=0.1$ ).

\section{Synthesis of the Poly(aldehyde guluronate) (PAG)}

PAG was synthesized according to a previously reported procedure. ${ }^{29}$ Briefly, sodium alginate was hydrolyzed under acidic conditions and the polyguluronic acid oligomers were isolated at $\mathrm{pH}$ 2.85. Polyguluronic acid was then dissolved in wa- 
ter with the addition of aqueous sodium hydroxide. Sodium polyguluronate was then precipitated with ethanol, filtered, and dried under reduced pressure; MW $6200 \mathrm{Da} ; M_{\mathrm{w}} / M_{\mathrm{n}} 1.14$; FTIR (KBr pellet, $\mathrm{cm}^{-1}$ ) 3430.5, 2931.2, 1616.4, 1412.5, $1320.5,1126,1092,1028 ;{ }^{1} \mathrm{H}$ NMR (360 MHz, $\mathrm{D}_{2} \mathrm{O}, \delta$ ): 3.90 (br, s), 3.99 (br, s), 4.07 (br, s), 4.45 (br, s), 5.03 (br, s); ${ }^{13} \mathrm{C}$ NMR (360 $\left.\mathrm{MHz}, \mathrm{D}_{2} \mathrm{O}, \delta\right)$ : $67.64,69.87,71.73,82.94,103.66,178.29$. An aqueous solution of sodium polyguluronate was then oxidized with sodium periodate in the dark at ambient temperature. The product was purified by extensive dialysis (Molecular Weight Cut Off (MWCO 1000)) against double-distilled water for 3 days. The solution was concentrated, frozen, and lyophilized to yield PAG; MW $6000 \mathrm{Da}, M_{\mathrm{w}} /$ $M_{\mathrm{n}}$ 1.4; FTIR (KBr pellet, $\mathrm{cm}^{-1}$ ) 3336.5, 2942, $1724,1616.4,1405.8,1321.1,1159.4,1117.2$, 1025.8. The aldehyde content was quantified using trinitrobenzene sulfonic acid. ${ }^{29}$

\section{Preparation of Covalent Cross-Linked PAG Hydrogels}

Daunomycin hydrochloride ( $10 \mu \mathrm{L}, 25 \mathrm{mg} / \mathrm{mL}$ solution in DMSO) was added to aqueous solutions of adipic dihydrazide $(25-125 \mu \mathrm{L}, 0.5 \mathrm{M})$ in $5-\mathrm{mL}$ sterile tubes. After $10 \mathrm{~min}$, aqueous solutions of PAG (150-200 $\mu \mathrm{L}, 20 \% \mathrm{w} / \mathrm{w})$ were added and the total volume was diluted to $500 \mu \mathrm{L}$ with Dulbecco's Modified Eagle's Medium (DMEM, pH 7.4) containing penicillin and streptomycin (100 units/ $\mathrm{mL}$ and $100 \mu \mathrm{g} / \mathrm{mL}$, respectively). The solutions were mixed thoroughly for $30 \mathrm{~s}$ and allowed to stand at room temperature for $1 \mathrm{~h}$ to form hydrogels. In the Results section of this manuscript, hydrogels are described by their final PAG concentrations (\% w/w) and final adipic dihydrazide concentrations ( $\mathrm{mM})$.

\section{Preparation of Ionic and Covalent Cross-Linked PAG Hydrogels}

Daunomycin hydrochloride $(10 \mu \mathrm{L}, 25 \mathrm{mg} / \mathrm{mL}$ solution in DMSO) was added to aqueous solutions of adipic dihydrazide $(25-125 \mu \mathrm{L}, 0.5 \mathrm{M})$ in 5 -mL sterile tubes. After $10 \mathrm{~min}$, aqueous calcium chloride $(20 \mu \mathrm{L}, 1 \mathrm{M})$ was added followed by aqueous solutions of PAG (150-200 $\mu \mathrm{L}, 20 \% \mathrm{w} / \mathrm{w})$. The total volume was adjusted to $500 \mu \mathrm{L}$ with DMEM and the gels were formed as already described.

\section{Determination of the Swelling Ratio of the Hydrogels}

Hydrogels were formed at various concentrations of PAG, adipic dihydrazide, and calcium chloride in 24-well plates. The hydrogels were immersed in DMEM (pH 7.4) and incubated at $37^{\circ} \mathrm{C}$ for 24 $\mathrm{h}$ to reach the equilibrium swelling. The hydrogels were transferred to $2-\mathrm{mL}$ vials and weighed (wet weight). The gels were then frozen and lyophilized, and the dried samples were weighed (dry weight). The swelling ratio was defined as the ratio (wet weight - dry weight)/(dry weight). We have corrected for the contribution of amino acids and salts in DMEM by subtracting this value from the weights of the wet and dry gels.

\section{Determination of the Incorporated Daunomycin}

To quantify the amount of daunomycin that is incorporated in the gels, two sets of hydrogels were formed as already described. One set was loaded with daunomycin hydrochloride $(0.20 \mathrm{mg}$, $0.355 \mu \mathrm{mol})$ and the other with trypan blue $(0.32$ $\mathrm{mg}, 0.333 \mu \mathrm{mol})$. After gelation, the hydrogels were frozen and lyophilized. The dried solid in each tube was crushed and $2 \mathrm{~mL}$ of doubledistilled water was added. The solutions were then sonicated for $30 \mathrm{~min}$, quantitatively transferred to 5-mL volumetric flasks, and diluted with double-distilled water. Each solution was filtered through a $0.22-\mu \mathrm{m}$ filter, and the concentration of the dissolved drug was quantified spectrophotometrically at $480 \mathrm{~nm}$ for daunomycin and $588 \mathrm{~nm}$ for trypan blue.

\section{In Vitro Release of Daunomycin in Fresh Medium}

Hydrogels were formed in $15-\mathrm{mL}$ sterile tubes as already described. Aqueous solutions of $5 \mathrm{~mL}$ of DMEM containing penicillin and streptomycin were added to each gel. The hydrogels were then incubated at $37^{\circ} \mathrm{C}$, and the medium was replaced with fresh DMEM (5 mL) periodically. The concentration of the released drug in the medium was quantified spectrophotometrically at $480 \mathrm{~nm}$.

\section{In Vitro Release of Daunomycin in Spent Medium}

Spent media was obtained from cultured KB cells. Approximately $2 \times 10^{4}$ cells were seeded in a 162 $\mathrm{cm}^{2}$ flasks containing $50 \mathrm{~mL}$ of media and incubated at $37^{\circ} \mathrm{C}$ for 3 to 4 days until cells had approximately reached confluency. The $\mathrm{pH}$ of the collected media was not significantly different from fresh media ( $\mathrm{pH}$ 7.4). Hydrogels containing daunomycin (10 $\mu \mathrm{L}, 25 \mathrm{mg} / \mathrm{mL}$ in DMSO) were 
formed at $6 \% \mathrm{w} / \mathrm{w}$ PAG and $150 \mathrm{mM}$ adipic dihydrazide. The hydrogels were exposed to $2.5 \mathrm{~mL}$ of spent Minimal Essential Media with Hanks' salts $(\mathrm{MEMH})$ without phenol red that contained 10\% calf serum and penicillin/streptomycin (100 units/ $\mathrm{mL}$ and $100 \mu \mathrm{g} / \mathrm{mL}$, respectively). The medium was replaced weekly, and the concentration of daunomycin that was released from the hydrogels in spent media was determined at $480 \mathrm{~nm}$.

\section{Cytotoxicity Assay with KB Cells}

The inhibitory concentrations of the released daunomycin that killed $50 \%$ of the cells $\left(\mathrm{IC}_{50}\right)$ were determined using an established cytotoxicity assay with $\mathrm{KB}$ cells as described previously. ${ }^{30} \mathrm{Hy}-$ drogels at a final concentration of $6 \% \mathrm{w} / \mathrm{w}$ PAG and $150 \mathrm{mM}$ adipic dihydrazide containing $250 \mu \mathrm{g}$ of daunomycin were prepared and incubated in 5 $\mathrm{mL}$ of sterile media at $37{ }^{\circ} \mathrm{C}$. The media was tested and replaced with fresh media $(5 \mathrm{~mL})$ periodically. As a control, a set of hydrogels were not loaded with the drug and treated in the same fashion as just described. The KB cells were cultured and seeded at a density of $5 \pm 10^{3}$ cells/well in 96-well plates with a total volume of tissue culture medium of $200 \mu \mathrm{L} /$ well. Cells were allowed to attach for 1 day before adding drug solutions or aliquots from the collected samples. Eight dilutions were used for each sample (each being onethird the concentration of the previous dilution). After $48 \mathrm{~h}$, cells were fixed with $95 \%$ ethanol for 5 min, rinsed with tap water, and stained with 2.5 $\mathrm{mM}$ crystal violet in $20 \%$ aqueous methanol for 5 $\mathrm{min}$. The plates were rinsed again with tap water and $150 \mu \mathrm{L} /$ well acidified ethanol $(0.01 \mathrm{M} \mathrm{HCl})$ was added to elute the dye from stained cells. The concentration of the dye in each well was measured spectrophotometrically at $570 \mathrm{~nm}$. Doseresponse curves were generated by plotting percent inhibition ([sample absorbance/control absorbance] $\times 100$ ) versus the log of the daunomycin concentration and fit to a line. The $\mathrm{IC}_{50}$ values were calculated using the equations for the best linear fit. $\mathrm{The} \mathrm{IC}_{50}$ values of daunomycin released from the hydrogels exposed to both fresh and spent media were compared with those of daunomycin stored in DMSO or daunomycin conjugated with adipic dihydrazide for 15 min before adding to the cells. Solutions containing only PAG, adipic dihydrazide, or material released from the hydrogels without daunomycin were also tested.

\section{RESULTS AND DISCUSSION}

\section{Characteristics of Poly(aldehyde guluronate) Hydrogels}

Commercially available high molecular weight alginate was hydrolyzed under acidic conditions to breakdown the $\beta$-glycosidic linkages between the mannuronate and guluronate residues as previously described. ${ }^{29}$ Polyguluronic acid was then precipitated at $\mathrm{pH} 2.85$, filtered, and reconstituted in water by adjusting the $\mathrm{pH}$ to 7 with sodium hydroxide to yield sodium polyguluronate. Sodium polyguluronate was then oxidized with sodium periodate to yield the PAG. The degree of oxidation was controlled by the mole equivalent of sodium periodate used in each reaction. The degree of oxidation of PAG used in this study was 87 $\pm 1 \%{ }^{29}$ The molecular weight and polydispersity of PAG did not significantly differ from the starting sodium polyguluronate. The average number of aldehyde groups per chain was estimated to be 67 groups. PAG was subsequently cross-linked with a bifunctional cross-linker, adipic dihydrazide, to form hydrogels (Figure 1). A high efficiency of this coupling was previously confirmed using Fourier transform-infrared (FT-IR) spectra and quantification of the cross-link density. ${ }^{29,31}$ The degree of swelling of PAG hydrogels was analyzed after the hydrogels had reached the equilibrium swelling in aqueous DMEM. The swelling ratio ranged from 15.5 to10.5 for hydrogels made with varying extents of cross-linking (Table 1). As expected, increasing the cross-link density resulted in a decrease in the water content in these hydrogels. The swelling was minimally affected by alterations in the concentration of the polymer used in this study. This finding may be attributed to the low molecular weight of the PAG chains utilized to form these hydrogels.

\section{Daunomycin Incorporation}

Drugs from the anthracyclin family had been previously incorporated into polymeric carriers for systemic drug delivery via the formation of hydrazone bonds. ${ }^{32,33}$ The ketone group on the C13 position of these drugs reacts with pendant hydrazide groups on the polymer backbone. A similar reaction was utilized in this paper to incorporate daunomycin into hydrogels for localized drug delivery. Aqueous solutions of daunomycin and adipic dihydrazide were mixed for $10 \mathrm{~min}$ before the addition of aqueous PAG solutions. During this time, daunomycin reacts with adipic dihydra- 


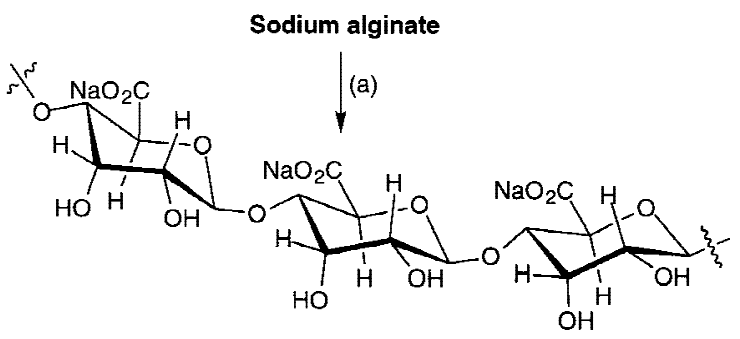

Sodium poly(guluronate)

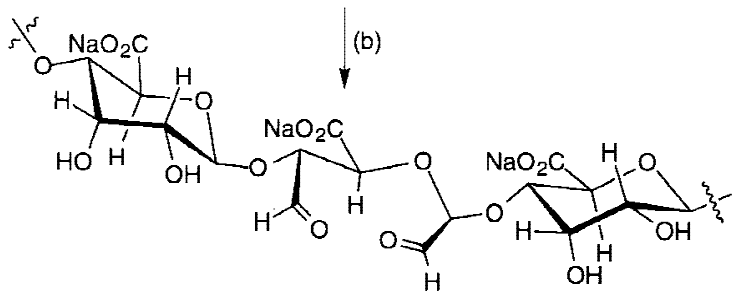

Poly(aldehyde guluronate)

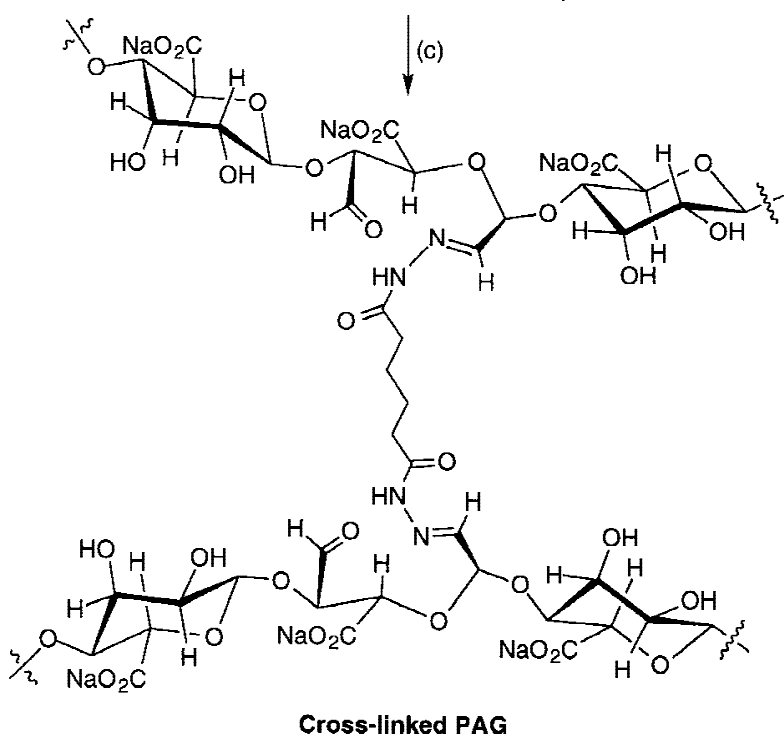

Figure 1. Synthesis of cross-linked poly(aldehyde guluronate) hydrogels: (a) reflux in aqueous hydrochloric acid followed by neutralization; (b) aqueous sodium periodate; and (c) aqueous adipic dihydrazide.

zide to form the daunomycin-adipoyl hydrazide conjugate. In theory, one hydrazide terminal of adipic dihydrazide reacts with the drug to form a hydrazone bond, whereas the other terminal is free because a large excess amount of adipic dihydrazide was used (Figure 2). The free hydrazide group of the daunomycin-adipoyl hydrazide conjugate potentially reacts with an aldehyde group on the backbone of PAG to form a labile hydrazone bond. The rest of the adipic dihydrazides potentially cross-link PAG to form hydrogels.

Daunomycin hydrochloride and adipic dihydra-
Table 1. Degree of Swelling of PAG Hydrogels ${ }^{a}$

\begin{tabular}{cccc}
\hline $\begin{array}{c}\text { PAG } \\
(\% \text { w/w })\end{array}$ & $\begin{array}{c}\text { Adipic dihydrazide } \\
(\mathrm{mM})\end{array}$ & $\begin{array}{c}\mathrm{CaCl}_{2} \\
(\mathrm{mM})\end{array}$ & Swelling ratio \\
\hline 6.0 & 100 & 40 & $15.4 \pm 0.4$ \\
6.0 & 150 & 40 & $11.3 \pm 0.1$ \\
6.0 & 200 & 40 & $11.0 \pm 0.1$ \\
6.0 & 250 & 40 & $10.6 \pm 0.2$ \\
6.0 & 150 & 0 & $14.2 \pm 0.2$ \\
7.0 & 150 & 0 & $13.3 \pm 0.2$ \\
8.0 & 150 & 0 & $12.8 \pm 0.3$ \\
\hline
\end{tabular}

${ }^{a}$ Hydrogels were prepared under various conditions; hydrogels were immersed in DMEM ( $\mathrm{pH} 7.4)$ and incubated at $37^{\circ} \mathrm{C}$ for $24 \mathrm{~h}$.

zide were mixed in water to form daunomycinadipoyl hydrazide. The reaction was monitored with TLC. After 15 min incubation at room temperature, unreacted free daunomycin could be detected at all concentrations of adipic dihydrazide (50-250 mM). The reaction was complete after $6 \mathrm{~h}$ of incubation at room temperature. HPLC was then used to quantitate the amount of daunomycin that reacted with adipic dihydrazide before the addition of PAG. Daunomycin hydrochloride and adipic dihydrazide were mixed at room temperature and analyzed 15 min later. The mixture

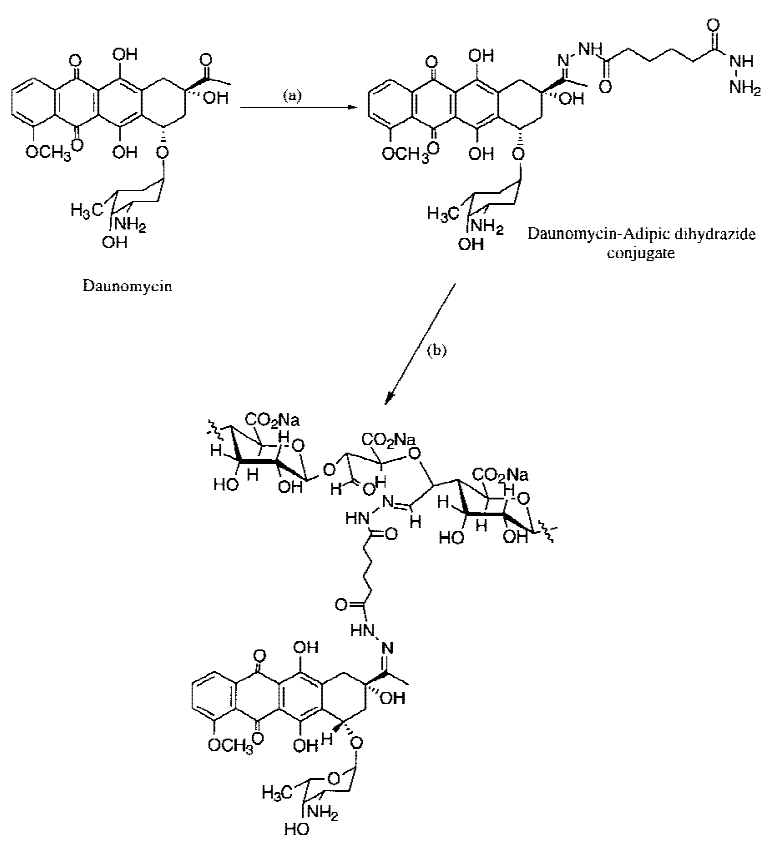

Figure 2. Scheme for the coupling of daunomycin to poly(aldehyde guluronate) hydrogels: (a) aqueous adipic dihydrazide; and (b) aqueous PAG. 
contained $88.5 \% \pm 0.5$ of the daunomycin-adipoyl hydrazide conjugate and $11.5 \% \pm 0.4$ of free drug (Figure 3). The amount of daunomycin that was covalently incorporated into the hydrogel was next determined by quantifying the amount of free drug in the hydrogel. Hydrogels containing daunomycin hydrochloride or trypan blue were synthesized, frozen, lyophilized, and crushed to form powders. The free drug was then dissolved

(a)

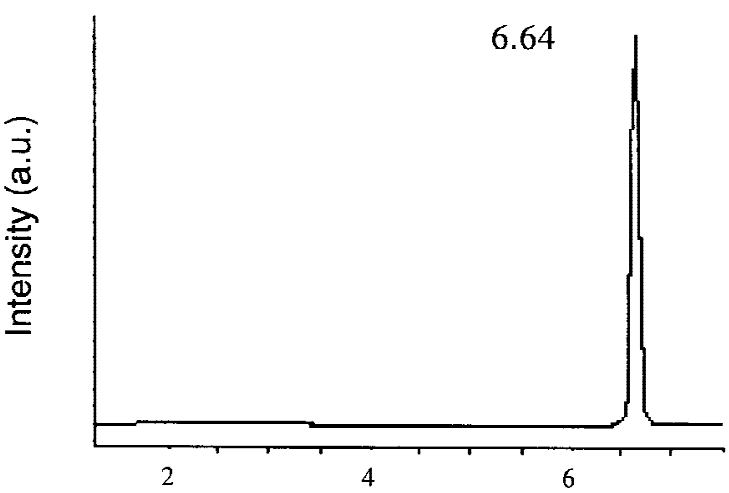

(b)

(c)

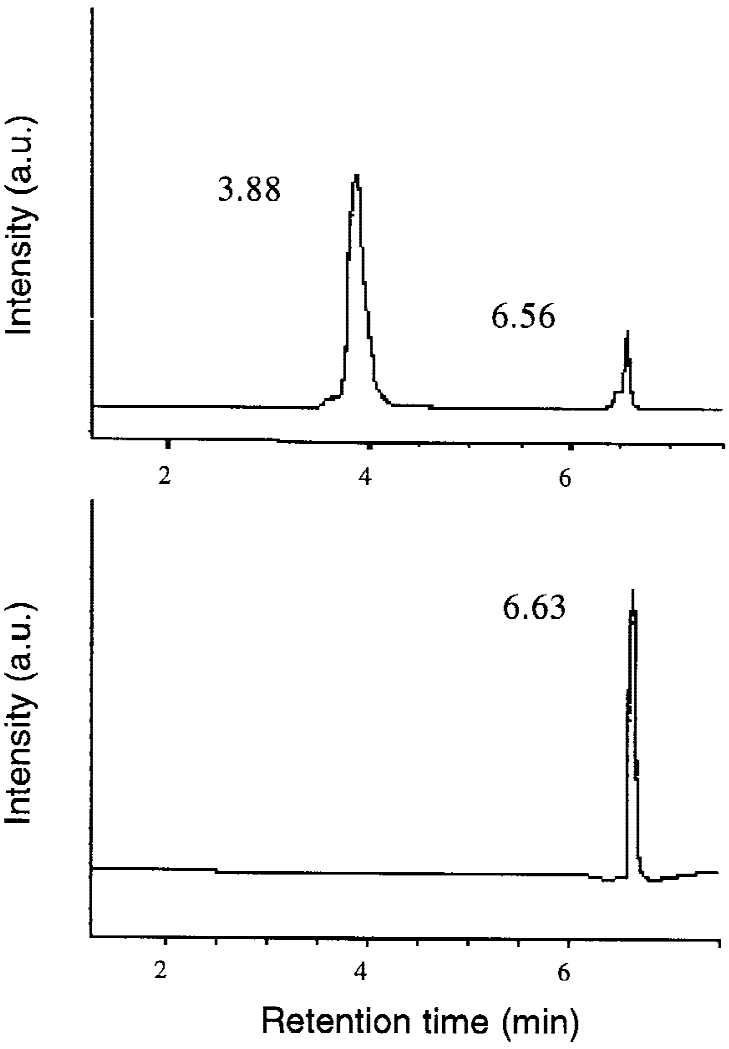

Figure 3. Reversed-phase (Hypersil C18) liquid chromatographic analysis of (a) daunomycin, (b) daunomycin-adipoyl hydrazide, and (c) daunomycin released from PAG hydrogels. The mobile phase consisted of $\mathrm{H}_{2} \mathrm{O} / \mathrm{CH}_{3} \mathrm{CN}(82 / 18$, v/v) and the aqueous component contained $0.025 \% \mathrm{v} / \mathrm{v}$ acetic acid. in water and the resulting mixture was filtered to remove any fine particles. The concentration of the free drug was then determined spectrophotometrically. Trypan blue was used as a control because it does not contain aldehyde or ketone groups for coupling to PAG and has a comparable molecular weight to daunomycin. Only $2 \%$ of the trypan blue was retained in the hydrogel. In comparison, $86.9 \pm 0.1 \%$ of daunomycin remained in the gelatinous particles. Similar results were obtained when ethanol was used to extract daunomycin and trypan blue. Moreover, hydrogels containing trypan blue that were incubated in DMEM released the entire drug within $24 \mathrm{~h}$ (data not shown). To eliminate the possibility that ionic interactions between the carboxylate groups on the polymer backbone and the ammonium group on the sugar ring of daunomycin control drug release, the release of daunomycin and daunomyci$\mathrm{n}$-adipoyl hydrazide conjugate from sodium alginate hydrogels was investigated. Alginate hydrogels cross-linked with calcium released the total loaded drug and conjugate within $24 \mathrm{~h}$ in DMEM (data not shown). The alginate hydrogels used in this study exhibited the swelling ratios of $33.5 \pm$ 1.9 and $46.9 \pm 1.2$ before and after drug release, respectively. These findings clearly indicate that daunomycin is covalently coupled to the PAG hydrogel through the adipic dihydrazide molecule. The daunomycin incorporation in the hydrogels correlates well with the percentage of daunomycin-adipic dihydrazide conjugate that was determined by HPLC analysis.

\section{In Vitro Release}

The release of the free drug from the hydrogel may be controlled by the chemical hydrolysis of the hydrazone bond between the drug and the adipic dihydrazide spacer, followed by the diffusion of the drug from the hydrogel. ${ }^{34}$ However, the hydrolysis of the linkage between adipic dihydrazide and PAG could also release the daunomycin-adipoyl hydrazide conjugate. In this case, an inactive form of the drug would be released (prodrug), which would be later activated by the hydrolysis of the linkage between adipic dihydrazide and daunomycin. To determine the percentage of free drug that is released, samples were periodically analyzed by HPLC. Uncoupled free daunomycin was quantitatively released from crosslinked PAG hydrogels at all times (Figure 3c). These results suggest that hydrolysis of the hy- 
drazone linkage between drug and adipic dihydrazide is the dominant reaction in drug release.

In the next series of experiments hydrogels were formed with daunomycin while varying the concentrations of adipic dihydrazide, calcium ions, and PAG. The release profile of the incorporated daunomycin from the hydrogel changed significantly depending on the concentration of adipic dihydrazide, calcium ions in the hydrogel, and PAG. In the first experiment, $6 \% \mathrm{w} / \mathrm{w}$ PAG hydrogels at various concentration of adipic dihydrazide were prepared. Hydrogels cross-linked at $50 \mathrm{mM}$ of adipic dihydrazide released the loaded drug completely in 2 days (Figure 4). As the concentration of the cross-linker was increased, the release rate of daunomycin was decreased. All incorporated drug was released in 2 weeks at 100 $\mathrm{mM}$ adipic dihydrazide, and in $>6$ weeks at elevated concentrations of adipic dihydrazide. It is important to note that at low concentrations of adipic dihydrazide, the complete release of daunomycin was accompanied by the complete dissolution of the hydrogel. Hydrogels with low crosslink density degrade at a faster rate (unpublished results).

Ionic cross-linking of PAG with calcium ions contributes to the physical properties of crosslinked PAG hydrogels in a similar manner as observed with alginate. ${ }^{29}$ The calcium ions cross-

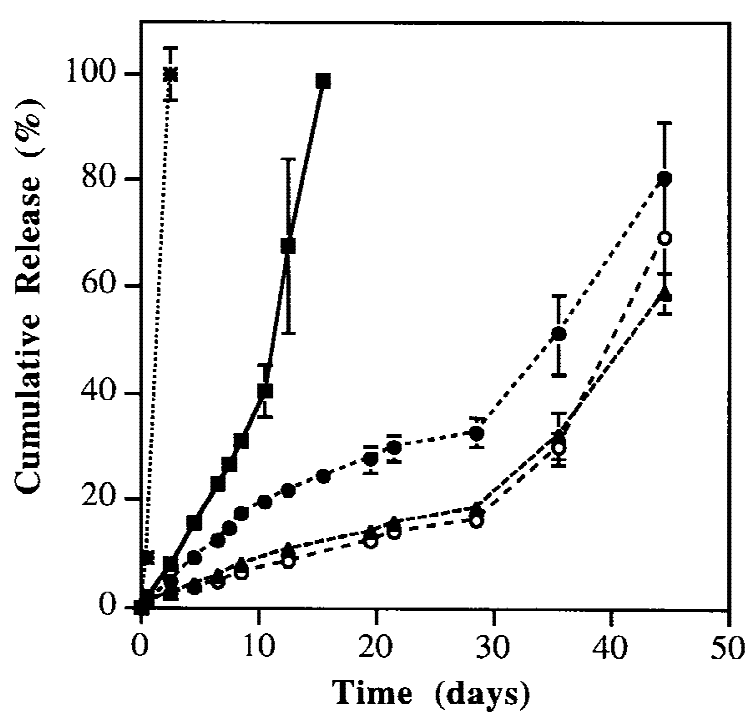

Figure 4. The cumulative release of daunomycin over time from hydrogels cross-linked by $50 \mathrm{mM}$ (*), 100 $\mathrm{mM}(\boldsymbol{\square}), 150 \mathrm{mM}(\bullet), 200 \mathrm{mM}(\boldsymbol{\Delta})$, and $250 \mathrm{mM}(\bigcirc)$ of adipic dihydrazide. All hydrogels were formed with $6 \%$ w/w PAG and $20 \mathrm{mM} \mathrm{CaCl}_{2}$, and release was monitored following incubation in DMEM $(\mathrm{pH} 7.4)$ at $37^{\circ} \mathrm{C}$.

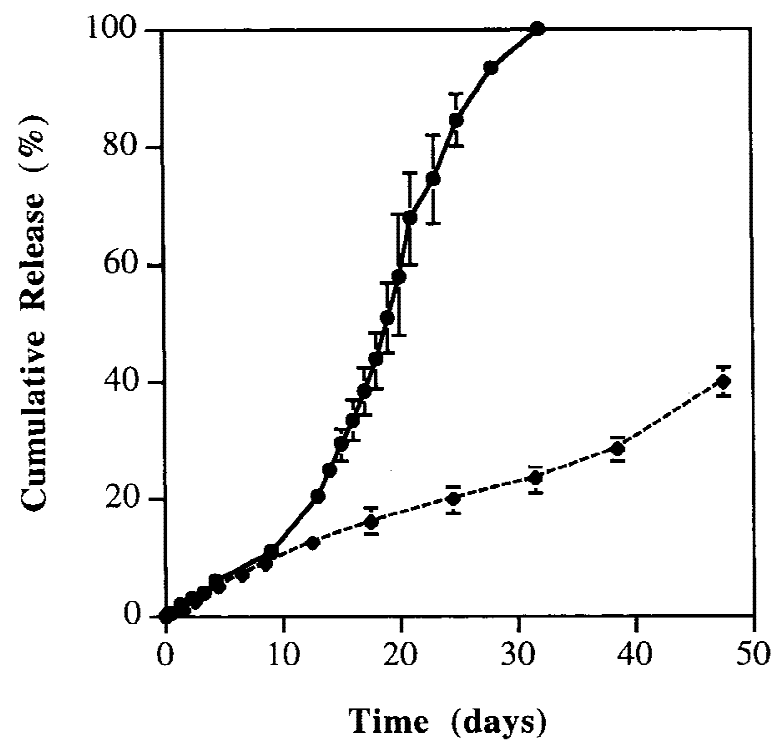

Figure 5. The cumulative release of daunomycin over time from 6\% w/w PAG hydrogels cross-linked by 150 $\mathrm{mM}$ adipic dihydrazide in the absence of calcium chloride (O) and in the presence of $40 \mathrm{mM}$ calcium chloride $(\diamond)$. Release was monitored following incubation in DMEM (pH 7.4) at $37{ }^{\circ} \mathrm{C}$.

link the carboxylate groups on adjacent PAG strands. To investigate the effect of calcium crosslinking on the release profile of daunomycin, aqueous solutions of $6 \% \mathrm{w} / \mathrm{w}$ PAG containing 250 $\mu \mathrm{g}$ daunomycin hydrochloride were cross-linked at $150 \mathrm{mM}$ adipic dihydrazide. In the absence of calcium ions in the hydrogels, a linear release of $1 \%$ of the incorporated drug per day was observed during the first 10 days followed by $\sim 4 \%$ release per day for the following 22 days (Figure 5). The total incorporated drug was released in 32 days. It is important to note that calcium ions are present in the medium at physiological concentrations in these studies. In the presence of $40 \mathrm{mM} \mathrm{CaCl}_{2}$ in the hydrogel, only $40 \%$ of the incorporated drug was released during the first 6 weeks, and it was released at a rate of $0.8 \%$ per day. The calcium cross-linking results in hydrogels with a high cross-linking density and a decreased pore size, which likely decreased the release of the drug from the hydrogel.

The extracellular $\mathrm{pH}$ of most tumors is slightly lower than that of normal tissues ${ }^{35,36}$ which could affect the release kinetics of drugs from PAG hydrogels. To investigate the possible role of $\mathrm{pH}$ on drug release, the release of daunomycin from $6 \%$ w/w PAG hydrogels at $150 \mathrm{mM}$ adipic dihydrazide was monitored at different $\mathrm{pH}$ conditions (Figure 
6). Daunomycin was released at a rate of $1 \%$ per day from hydrogels incubated at neutral condition, but daunomycin was released at a significantly higher rate (1.8\% per day) from hydrogels incubated in acidic medium ( $\mathrm{pH} 6)$. This result is likely caused by the higher rate of hydrolysis of hydrazone bonds under acidic conditions. ${ }^{37,38}$ The minimal further change in the release as the $\mathrm{pH}$ was further lowered to $\mathrm{pH} 5$ could be related to several factors. The change in hydrolysis from $\mathrm{pH}$ 6 to 5 may not be significant. Alternatively, the hydrogel is polyanionic and as the $\mathrm{pH}$ decreases, a higher percentage of the carboxyl groups are protonated. This situation may cause changes in the gel structure that alter the drug diffusion (e.g., changing pore size in gel).

\section{Antitumor Activity of Released Daunomycin}

The antitumor activity of the daunomycin released from PAG hydrogels was evaluated in vitro using a standard cytotoxicity assay with $\mathrm{KB}$ cells. ${ }^{29}$ The media collected from control hydrogels had no effect on cell growth. The $\mathrm{IC}_{50}$ of the daunomycin-adipoyl hydrazide conjugate (in the presence of 100-fold excess of adipic dihydrazide) was $0.224 \pm 0.091 \mu \mathrm{M}$ compared with $0.202 \pm$ $0.048 \mu \mathrm{M}$ for the free daunomycin (Table 2). The activity of the released daunomycin slightly de-

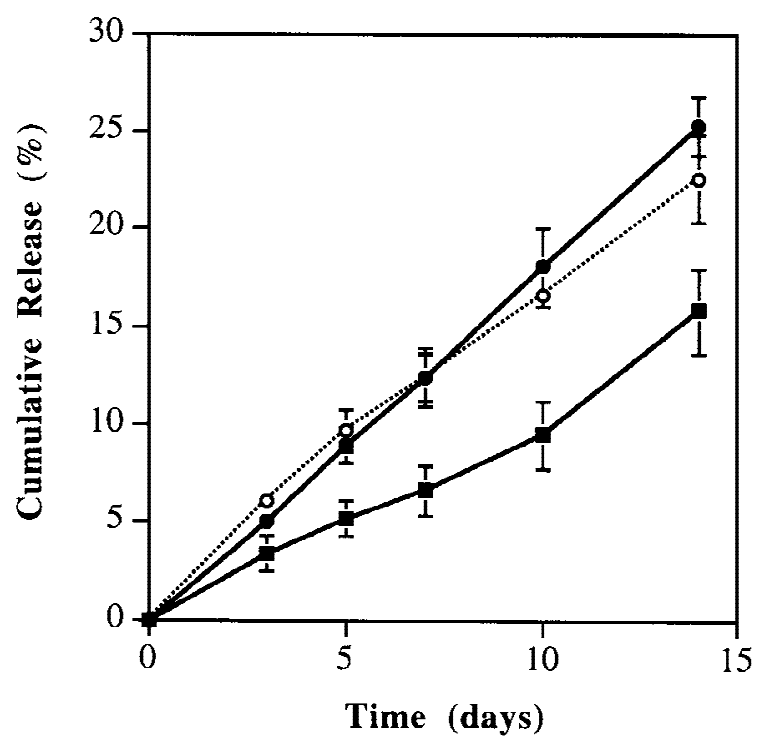

Figure 6. The cumulative release of daunomycin over time at pH $5(\mathbf{O})$, pH $6(\bigcirc)$, and pH $7.4(\mathbf{\square})$. All hydrogels were formed with $6 \%$ w/w PAG and $150 \mathrm{mM}$ adipic dihydrazide and release was monitored following incubation in DMEM at $37^{\circ} \mathrm{C}$.
Table 2. Inhibitory Activity of Intact or Released Daunomycin Towards KB Cells over Time

\begin{tabular}{lc}
\hline \multicolumn{1}{c}{ Sample } & $\mathrm{IC}_{50}(\mu \mathrm{M})$ \\
\hline Free daunomycin & $0.202 \pm 0.048$ \\
Daunomycin-adipic dihydrazide & $0.224 \pm 0.091$ \\
Released daunomycin after 1 week & $0.560 \pm 0.149$ \\
Released daunomycin after 2 weeks & $0.349 \pm 0.176$ \\
Released daunomycin after 3 weeks & $0.805 \pm 0.229$ \\
\hline
\end{tabular}

creased during the first 3 weeks, suggesting that the released drug might be partially degraded. The glycosidic linkage between the daunosamine and daunomycinone units of the drug has been previously reported to undergo hydrolytic cleavage under neutral to basic conditions. ${ }^{39,40}$ The activity of daunomycin released in spent media did not differ significantly from that released in fresh media (data not shown). PAG and adipic dihydrazide were also tested to ensure that the noted cytotoxicity was not caused by these components of the hydrogels. Neither aqueous PAG nor adipic dihydrazide had a significant effect on the growth of $\mathrm{KB}$ cells over the range of concentrations tested (Table 2).

\section{CONCLUSIONS}

We have developed a novel biodegradable hydrogel for the sustained local delivery of daunomycin over time frames ranging from a few days to several weeks. We propose that cross-linked poly(aldehyde guluronate) hydrogels loaded with daunomycin or other antineoplastic agents (containing aldehyde or ketone groups) can deliver these agents in vivo in a sustained manner. This system may provide a potent vehicle for the localized delivery of antineoplastic agents in a minimally invasive manner.

\section{ACKNOWLEDGMENTS}

The authors thank Reprogenesis for financial support of this research. The authors also thank Dr. John Drach and Julie Breitenbach for their kind help in the biological evaluations of daunomycin. GMK also gratefully acknowledges the support of a Medical Scientist Training Program Fellowship. 


\section{REFERENCES}

1. Lowenthal,RM, Eaton K. 1996. Toxicity of chemotherapy. Hematol Oncol Clin N 10:967-990.

2. Klein-Szanto AJ.1992. Carcinogenic effects of chemotherapeutic compounds. Prog Clin Biol Res 374: 167-174.

3. Jeyanthi R, Nagarajan B, Rao KP. 1991. Solid tumor-chemotherapy using implantable collagenpoly(HEMA) hydrogel containing 5-fluorouracil. J Pharm Pharmacol 43:60-62.

4. Francois JJ, Fortier G. 1996. Immobilization of Lasparaginase into a biocompatible poly(ethylene glycol)-albumin hydrogel. 1. Preparation and in vitro characterization. Biotech Appl Biochem 23: 221-226.

5. Putnam D, Kopeček J.1995. Polymer conjugates with anticancer activity. Adv Polym Sci 122: 55-123.

6. Yokoyama M, Fukushima S, Uehara R, Okamoto K, Kataoka K, Sakurai Y, Okano T. 1998. Characterization of physical entrapment and chemical conjugation of adriamycin in polymeric micelles and their design for in vivo delivery to a solid tumor. J Controlled Release 50:79-92.

7. Jaroszeski MJ, Gilbert R, Heller R. 1997. Electrochemotherapy: An emerging drug delivery method for the treatment of cancer. Adv Drug Deliv Rev 26:185-197.

8. Kwon G, Suwa S, Yokoyama M, Okano T, Sakurai Y, Kataoka K. 1994. Enhanced tumor accumulation and prolonged circulation times of micelleforming poly(ethylene oxide-aspartate) block copolymer-adriamycin conjugates. J Controlled Release 29:17-23.

9. Uchegbu IF, Duncan R. 1997. Niosomes containing $\mathrm{N}$-(2-hydroxypropyl)methacrylamide copolymerdoxorubicin (PK1): Effect of method of preparation and choice of surfactant on niosome characteristics and a preliminary study of body distribution. Int $\mathrm{J}$ Pharm 155:7-17.

10. Kratz F, Beyer U, Roth T, Tarasova N, Collery P, Lechenault F, Cazabat A, Schumacher P, Unger C, Falken U. 1998. Transferrin conjugates of doxorubicin: Synthesis, characterization, cellular uptake, and in vitro efficacy. J Pharm Sci 87:338-346.

11. ̌̌́íhová B, Strohalm J, Plocová D, Šubr V, Šrogl J, Jelínková M, Śírová M, Ulbrich K. 1996. Cytotoxic and cytostatic effects of anti-Thy 1.2 targeted doxorubicin and cyclosporin A. J Controlled Release 40: 303-319.

12. Pouyani T, Prestwich GD. 1994. Functionalized derivatives of hyaluronic acid oligosaccharides: Drug carriers and novel biomaterials. Bioconjugate Chem 5:339-347.

13. Jeong B-M, Bae YH, Lee DS, Kim SW. 1997. Biodegradable block copolymers as injectable drug delivery systems. Nature 388:860-861.

14. Patil NS, Dordick JS, Rethwisch DG. 1996. Macro- porous poly(sucrose acrylate) hydrogel for controlled release of macromolecules. Biomaterials 17: 2343-2350.

15. Hubbell JA. 1996. Hydrogel systems for barriers and local drug delivery in the control of wound healing. J Controlled Release 39:305-313.

16. Inoue $\mathrm{T}$, Chen GH, Nakamae K, Hoffman ASA 1997. Hydrophobically-modified bioadhesive polyelectrolyte hydrogel for drug delivery. J Controlled Release 49:167-176.

17. Zhao X, Harris JM. 1998. Novel degradable poly(ethylene glycol) hydrogels for controlled release of protein. J Pharm Sci 87:1450-1458.

18. Andreopolos FM, Dieble CR, Stauffer MT, Webber SG, Wagner WR, Beckman EJ, Russel AJ. 1996. Photoscissable hydrogel synthesis via rapid photopolymerization of novel PEG-based polymers in the absence of photoinitiators. J Am Chem Soc 118: 6235-6240.

19. Sutherland IW. 1991. Alginates. In: Byron D., editor Biomaterials: Novel materials from biological sources. New York: Stockton Press, pp 309-331.

20. Gombotz WR, Wee SF. 1998. Protein release from alginate matrices. Adv Drug Deliv Rev 31:267-285.

21. Shapiro L, Cohen S. 1997. Novel alginate sponges for cell culture and transplantation. Biomaterials 18:583-590.

22. Bouhadir KH, Mooney DJ. 1998. In vitro and in vivo models for the reconstruction of intercellular signaling. Ann NY Acad Sci 842:188-194.

23. Klock G, Pfeffermann A, Ryser C, Grohn P, Kuttler B, Hahn HJ, Zimmermann U. 1997. Biocompatibility of mannuronic acid-rich alginates. Biomaterials 18:707-713.

24. Skják-Braek G, Murano E, Paoletti S. 1989. Alginate as immobilization material. II: Determination of polyphenol contaminants by fluorescence spectroscopy, and evaluation of methods for their removal. Biotechnol Bioeng 33:90-94.

25. Kikuchi A, Kawabuchi M, Sugihara M, Sakurai Y, Okano T. 1997. Pulsed dextran release from calcium-alginate gel beads. J Controlled Release 47: 21-29.

26. Morgan SM, Al-Shamkhani A, Callant D, Schacht E, Woodley JF, Duncan R. 1995. Alginates as drug carriers: Covalent attachment of alginates to therapeutic agents containing primary amine groups. Int J Pharm 122:121-128.

27. Murata Y, Miyamoto E, Kawashima S.1996. Additive effect of chondroitin sulfate and chitosan on drug release from calcium-induced alginate gel beads. J Controlled Release 38:101-108.

28. Al-Shamkhani A, Duncan R. 1995. Radioiodination of alginate via covalently-bound tyrosinamide allows for monitoring of its fate in vivo. $\mathrm{J}$ Bioact Compat Polym 10:4-13.

29. Bouhadir KH, Hausman DS, Mooney DJ. 1999. 
Synthesis of cross-linked poly(aldehyde guluronate) hydrogels. Polymer 40:3575-3584.

30. Prichard MN, Prichard LE, Baguley WA, Nassiri MR, Shipman C. 1991. Three-dimensional analysis of the synergistic cytotoxicity of ganciclovir and zidovudine. Antimicrob Agents Chemother 35: 1060-1065.

31. Lee KY, Bouhadir KH, Mooney DJ. 2000. Degradation behavior of covalently cross-linked poly(aldehyde guluronate) hydrogels Macromolecules 33: 97-101.

32. Firestone RA, Willner D, Hofstead SJ, King HD, Kaneko T, Braslawsky GR, Greenfield RS, Trail PA, Lasch SJ, Henderson AJ, Casazza AM, Hellstrom I, Hellstrome KE. 1996. Synthesis and antitumor activity of immunoconjugate BR96-Dox. J Controlled Release 39:251-259.

33. Kratz F, Beyer U, Schumacher P, Kruger M, Zahn H, Roth T, Fiebig HH, Unger C. 1998. Transferrin conjugates of doxorubicin: synthesis, characterization, cellular uptake, and in vitro efficacy. J Pharm Sci 87:338-346.

34. Heindel ND, Zhao H, Leiby J, VanDongen JM, Lacey CJ, Lima DA, Shabsoug B, Buzby JH. 1990. Hydrazide pharmaceuticals as conjugates to polyaldehyde dextran: Syntheses, characterization, and stability. Bioconjugate Chem 1:77-82.
35. Tannock IF, Rotin D. 1989. Acid pH in tumors and its potential for therapeutic exploitation. Cancer Res 49:4373-4384.

36. Gerweck LE, Seetharaman K. 1996. Cellular pH gradient in tumor versus normal tissue: Potential exploitation for the treatment of cancer. Cancer Res 56:1194-1198.

37. Greenfield RS, Kaneko T, Daues A, Edson MA, Fitzgerald KA, Olech LJ, Grattan JA, Spitalny GL, Braslawsky GR. 1990. Evaluation in vitro of adriamycin immunoconjugates synthesized using an acid-sensitive hydrazone link. Cancer Res 50: 6600-6607.

38. Kaneko T, Willner D, Monkovic I, Knipe JO, Braslawsky GR, Greenfield RS, Vyas DM. 1991. New hydrazone derivatives of adriamycin and their immunoconjugates-A correlation between acid stability and cytotoxicity. Bioconjugate Chem 2:133-141.

39. Pujol M, Munoz M, Prat J, Girona V, DeBolos J. 1997. Stability study of epirubicin in $\mathrm{NaCl} 0.9 \%$ injection. Ann Pharmacother 31:992-995.

40. Nyhammar EK, Johansson SG, Seiving BE. 1996. Stability of doxorubicin hydrochloride and vincristine sulfate in two portable infusion-pump reservoirs. Am J Health-System Pharm 53:1171-1173. 